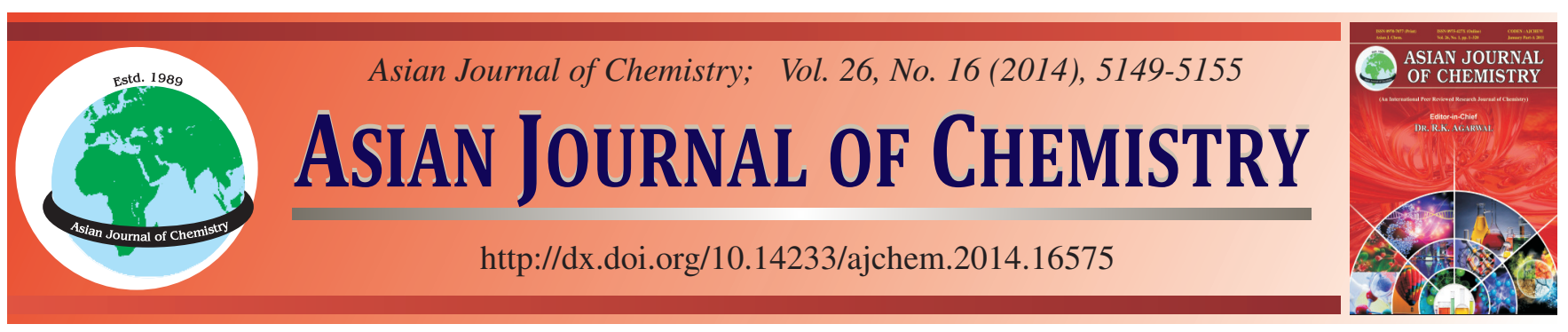

\title{
Simultaneous Determination of Seven Carbohydrates in Tobacco by Ultrasonic Extraction-Ion Chromatography
}

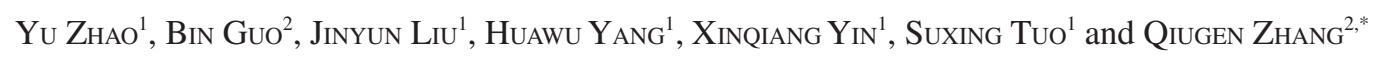

${ }^{1}$ Technology Center of China Tobacco Hunan Industrial Corporation, Changsha 410007, Hunan Province, P.R. China

${ }^{2}$ Key Laboratory of Jiangxi Province for Persistent Pollutants Control and Resources Recycle, Nanchang Hangkong University, Nanchang 330063, P.R. China

*Corresponding author: Fax: +86 791 8395373; Tel: +86 791 83953372; E-mail: niatzqg@163.com; fangfer@tom.com

A simple and reproducible method has been developed for the detection the carbohydrates of cigarettes. In this method, carbohydrates are obtained by ultrasonic extraction. The extracts are then analyzed using high-performance anion exchange chromatography followed by pulsed amperometric detection. The relative amounts of rhamnose, arabinose, fructose, glucose, xylose, maltose and sucrose, in cigarette are used for their characterization. The HPAEC-PAD method provided high selectivity, demonstrated good precision and had a large dynamic range in tobacco extracts.

Keywords: Pulsed amperometric detection, Tobacco, Chromatography, Carbohydrates.

\section{INTRODUCTION}

The identification and determination of carbohydrates are important research in tobacco industry, because it is intimately linked with the two hot topic 'enhancement flavor and maintaining moist' and 'reduction tar and damage' which are being discussed by researchers in tobacco industry. Nevertheless, the relationship between the two issues is very delicate and conditioned upon each other and they promote common development. Therefore, how to properly handle the relationship between the two issues becomes very significant ${ }^{1}$. The reduction of tar and damage are bound to reduce flue gas volume resulting fade smoke and worse taste. Thus flavors and fragrances should be added to improve the taste ${ }^{2}$. Carbohydrates are the main component of flavors and fragrances. However, a series of complex chemical reactions occur in the carbohydrates combustion process and the generated products are relative to the harmful components such as tar, $\mathrm{CO}$ and total particulate matter ${ }^{3,4}$. Most of the carbohydrates in cigarettes derive from tobacco leaf itself and a small amount of carbohydrates are added to the cigarettes by manufacturers. The amount of added sugars can be artificially controlled. But the amount of self-contained sugars in tobacco is influenced by the growth of regional environment, climate, tobacco varieties, the fabrication process and other relevant factors ${ }^{4}$. Therefore, establishing good analysis methods for analysis of carbohydrates is favorable to handle the relationship between the two issues, check the quality of tobacco and improve the recipe of tobacco.

Carbohydrates are usually hydrophilic, neutral and lack satisfactory chromophores for UV detection. Derivatization is often used to improve sensitivity and chromatographic resolution $^{5}$. In addition, these compounds do not give a persistent amperometric response for a constant applied potential at noble metal electrodes due to extensive fouling of the electrode surface. Refractive index (RI) detection, which is a bulk property detector and colorimetric methods for reducing sugars are not analyte specific and suffer from poor sensitivity ${ }^{6}$. On the other hand, direct, sensitive and reproducible detection of polar aliphatic compounds, such as carbohydrates, is accomplished by pulsed amperometric detection (PAD) at a gold electrode ${ }^{7}$. Unlike methods for reducing sugars, PAD is applicable to virtually all carbohydrates. High-performance anion exchange chromatography (HPAEC) is used for the separation of carbohydrates in alkaline media ${ }^{8}$. The technique HPAEC PAD is more precise, accurate and sensitive compared to other methods for the determination of sugars such as HPLC-RI. In addition, the possibility to a gradient elution increases the chance of a better resolution of chromatographic peaks in a short time, because of the matrix analyzed ${ }^{9}$. In this work we finally chose high performance anion exchange chromatography-pulsed amperometric detection (HPAEC-PAD) method to measure the main carbohydrates of tobacco and tobacco products in a without derivatization, easy to carry out, high selectivity and sensitivity and more green way. 


\section{EXPERIMENTAL}

ICS-3000 multifunctional ion chromatography (Dionex, America), including double pump (DP) module (one pump module and solvent delivery pump), detector/chromatograph (DC), automatic sampler (AS), Chromeleon 6.8 working station, Ampere detector use gold electrode as working electrode, $\mathrm{pH} /$ $\mathrm{Ag} / \mathrm{AgCl}$ as reference electrode, $\mathrm{Ti}$ as counter electrode; $\mathrm{AL}_{2} \mathrm{O}_{4}$ electronic scales(sensitive quality $0.0001 \mathrm{~g}$, Mettler Toledo, China); Milli-Q(Millipore, America); KQ-600DE ultrasonic cleaner (Kun Shan Ultrasonic Instruments Co., Ltd); Finnpipette (1-100 $\mu \mathrm{L}, 100-1000 \mu \mathrm{L}$, Eppendorf, Germany); OnGuard ${ }^{\circledR}$, RP(DIONEX, America); $0.45 \mu \mathrm{m}$ filter membrane (Dikma, China).

Ultrapure water, specific resistance $=18.2 \mathrm{MO} \mathrm{cm}^{-1}$, MilliQ); Glass instruments have been correction (correction factor 99.5-100.5\%) and then treat with water and Hydrochloric acid aqueous solution (v/v:1/1)24 h, dying.

Experimental sample: Cigarette samples have been sold in sealed plastic bags, kept at low temperature before use. Selected 10 packs of cigarettes by randomly, removed the filter and paper, take out the tobacco, mixed uniformly, put into $80{ }^{\circ} \mathrm{C}$ oven to drying and crush, through 400 mesh sieve and stored in brown plastic sealed and kept in the refrigerator. Unblended cigarette was suppoted by Changsha cigarette factory. Standards reagent can be seen in Table-1.

\begin{tabular}{lcc} 
& TABLE-1 \\
& STANDARDS REAGENT \\
\hline \multicolumn{1}{c}{ Reagent } & Purity $(\%)$ & Source \\
\hline L-(+)- rhamnose & 99 & $\begin{array}{c}\text { Alfa. Aesar, } \\
\text { Johnson Matthey }\end{array}$ \\
L-(+)- arabinose & 99 & Alfa. Aesar, \\
& & Johnson Matthey \\
D-(+)- fructose & $>8$ & TCI Shanghai \\
D-(+)- glucose & $>8$ & TCI Shanghai \\
D-(+)- xylose & $>8$ & TCI Shanghai \\
D-(+)- maltose & $>8$ & TCI Shanghai \\
D-(+)- sucrose & $>8$ & TCI Shanghai \\
Anhydrous sodium acetate & AR & GMP \\
NaOH & $50 \% \mathrm{w} / \mathrm{w}$ & Fisher scientific \\
\hline
\end{tabular}

Ion chromatography analysis: Aliquots of $10 \mu \mathrm{L}$ were analyzed on the Dionex ICS-3000 ion chromatograph equiped with a anion exchange column, Dionex AminoPac PA10 (2 mm i.d. $\times 50 \mathrm{~mm}$ ) and a guard column, Dionex AminoPac GA10 ( 2 mmi.d. $\times 250 \mathrm{~mm}$ ), which is consisted with a DP pump operating at a flow rate of $0.25 \mathrm{~mL} / \mathrm{min}$. The mobile phase was ultrapure water, $\mathrm{NaOH}(250 \mathrm{mmol} / \mathrm{L})$ and $\mathrm{NaAc}(1 \mathrm{~mol} /$ L). The column temperature was $30{ }^{\circ} \mathrm{C}$.

The elution mode was gradient elution and the program was showed in Table-2. Fig. 1 was obtained by integrated pulsed amperometry.

Pretreatment: Aliquots of $50 \mathrm{mg}$ of samples and $50 \mathrm{~mL}$ ultrapure water were added into a conical flask. The mixture of solution was under ultrasonic condition at $25^{\circ} \mathrm{C}$ and $40 \mathrm{~Hz}$ for $20 \mathrm{~min}$. The products obtained was put into refrigerator under $4{ }^{\circ} \mathrm{C}$ for further use. Before analysis, the samples were filtrated through $0.45 \mu \mathrm{m}$ filter membrane.

\begin{tabular}{cccccc}
\hline \multicolumn{5}{c}{ TABLE-2 } \\
GRADIENT ELUTION FOR THE \\
ANALYSIS OF WATER-SOLUBLE SUGARS \\
\hline Retention (min) & Flow (mL/min) & A (\%) & B (\%) & C $(\%)$ & Curve \\
\hline 0.00 & 0.250 & 90.8 & 9.2 & 0.0 & 5 \\
20.00 & 0.250 & 90.8 & 9.2 & 0.0 & 5 \\
26.00 & 0.250 & 64.0 & 36.0 & 0.0 & 8 \\
27.00 & 0.250 & 64.0 & 36.0 & 0.0 & 8 \\
30.50 & 0.250 & 40.0 & 20.0 & 40.0 & 8 \\
31.00 & 0.250 & 44.0 & 16.0 & 40.0 & 5 \\
33.00 & 0.250 & 14.0 & 16.0 & 70.0 & 8 \\
38.00 & 0.250 & 14.0 & 16.0 & 70.0 & 8 \\
38.10 & 0.250 & 20.0 & 80.0 & 0.0 & 5 \\
40.10 & 0.250 & 20.0 & 80.0 & 0.0 & 5 \\
40.20 & 0.250 & 90.8 & 9.2 & 0.0 & 5 \\
50.00 & 0.250 & 90.8 & 9.2 & 0.0 & 5 \\
\hline
\end{tabular}

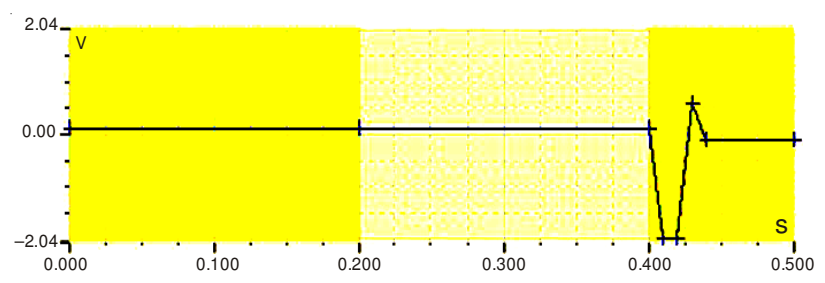

Fig. 1. Detection waveform for the analysis of water-soluble sugars

\section{RESULTS AND DISCUSSION}

Selection of detector and eluent: Carbohydrate is a kind of hydrophilic and weak acidic compounds, which exists in alkaline solution in the anionic form. Under strong alkaline environment, between the electrochemical activity groups (such as hydroxyl) of carbohydrate and gold electrode, redox reaction would occur on the surface of gold electrode under an appropriate potential, resulting in current change. Therefore, we can use pulse ampere detector to test the samples. Considering conditions, under which all kinds of carbohydrate were eluted through the anion analytical column, in present study, we select sodium hydroxide/sodium acetate as the mobile phase due to its strong elution ability.

Selection of waveform of detection potential: Ampere detection based on the four potential pulse waveform was used to test the samples due to its advantages of high sensitivity, good reproducibility and can eliminate sag on the surface of gold electrode and electrochemical corrosion phenomenon. In this paper, we finally choose point waveform to experiment as shown in Fig. 1.

Selection of column: In general, anion exchange column was selected to separate monosaccharide and disaccharide. We had selected CarboPacTMPA10, CarboPac TMPA20 and CarboPacTMPA100 to carry out contrasting test. Negative peak caused by dissolved oxygen had cause inconspicuous interference during the course of CarboPacTMPA10 test, showing good separation effect; Column capacity of CarboPacTMPA20 was low, can be used to implement fast separation, but the degree of separation was poor; Column capacity of CarboPacTMPA100 was Close to CarboPac TMPA10, but the effect of separation of fructose and sucrose was not good. According to the results of test, the separation effect of CarboPacTMPA10 was optimal, which was more economical 
and practical, in this study, we had selected CarboPac TMPA10 for subsequent experiment.

Selection of mobile phase ratio: Sodium hydroxide/ sodium acetate eluent system was selected as the mobile phase system due to its strong elution ability. Detector towards carbohydrate compounds produces high response under alkaline environment caused by hydroxyl playing important role in elution. Retention behaviour of carbohydrate in column was investigated through changing the concentration of sodium hydroxide. The result shows that the response of detector towards carbohydrate is not obvious under low concentration of sodium hydroxide, which was increasing with increasing concentration of sodium hydroxide. The retention time of carbohydrate will shorten at increasing concentration of sodium hydroxide, but the degree of separation will be correspondingly reduced. Reduction of the retention time of maltose was more obvious than others with the increase of the concentration of sodium hydroxide. The degree of separation of rhamnose and arabinose was good under high concentration of sodium hydroxide, which was less than 1 when the concentration of sodium hydroxide was under $20 \mathrm{mmol} / \mathrm{L}$. However, separation effect of xylose and aevulose was not good when the concentration of sodium hydroxide is higher than $50 \mathrm{mmol} / \mathrm{L}$. According to results, we finally selected elution condition showed in Table-2, under which the chromatogram of samples obtained (Fig. 2).

Optimization of eluent flow rate: The increasing flow rate can improve the efficiency of elution, but excessive velocity will reduce the degree of separation resulting in incomplete separation; Good separation degree can be obtained at low flow rate, but cause low elution efficiency, prolonged analysis and broad peaks. Furthermore, the high flow rate resulted in high system pressure. Beyond the pressure limit of the instrument, it may cause short lifetime of apparatus. After optimizing, $0.25 \mathrm{~mL} / \mathrm{min}$ was ultimately selected as eluent velocity.

Selection of column temperature: Different column temperature $\left(24,26,28,30\right.$ and $\left.32^{\circ} \mathrm{C}\right)$ were selected to analysis the same samples. The results showed that temperature had a little effect on the experimental results. Therefore we choose $30^{\circ} \mathrm{C}$ recommended by Diane company as working temperature. Finally, we found that the instrument system is relatively stable under $30^{\circ} \mathrm{C}$.

\section{Selection of sample pretreatment conditions}

Methods of extraction: A number of sugar extraction methods were already published. General means of sugar extraction in tobacco are ultrasonic extraction, shocking extraction, reflux extraction and direct soaking. Compared with above methods, we found that the shocking extraction was completed in atleast $1 \mathrm{~h}$ and $0.05 \mathrm{~h}$ atleast was taken to accomplish extraction by means of reflux extraction in the temperature of $80^{\circ} \mathrm{C}$, which, however, may lead to the decomposition of disaccharides and inaccurate results. To addition, it takes $24 \mathrm{~h}$ at least to completely extract by direct soaking. Nevertheless, only 20 min was satisfied to finish extraction by using ultrasonic extraction. Thus we select the method of ultrasonic extraction to extract surges from tobacco.

Selection of extraction solvent: Alcoholic solution and pure water were used to extract carbohydrate in the tobacco industry. Using alcohol solution as the extract solution easily causes interference peaks in the mode of amperometric detection. C.R. National Tobacco Board promulgated the "determination of the standard tobacco sugar" in 2002 which demands that using $5 \%$ acetic acid as extract solution. Sodium hydroxide solutions are often used as an extraction solution in Several references, which can also prevent hydrolysis ${ }^{10}$. In this article, we researched on using different solutions (the pure water, $5 \%$ acetic acid and $0.01 \mathrm{mmol} / \mathrm{L}$ sodium hydroxide aqueous) as the extraction solution to extract sugars from tobacco and the results were shown in Table-3, it shows that the evident optimum extraction solution is pure water. Thus we select pure water as a follow-up extraction solvent.

Selection liquid ratio: The content of different carbohydrate in tobacco is quite different. Determined solid-liquid ratio, all sugars should be extracted completely and the concentration of various sugars should be made at a suitable level. In this paper, ion chromatography methods for sugars were detected and the limit detection of the method is low. According to the above results, this paper compares the different concentration sample $(1,4$ and $10 \mathrm{mg} / \mathrm{L})$ extraction conditions. The results given in Table-4. The best concentration condition for extraction was $1 \mathrm{mg} / \mathrm{L}$.

Selection of ultrasonic extraction time: Firstly, aliquots of $300 \mathrm{mg}$ of samples divided equally into 6 parts. Each part

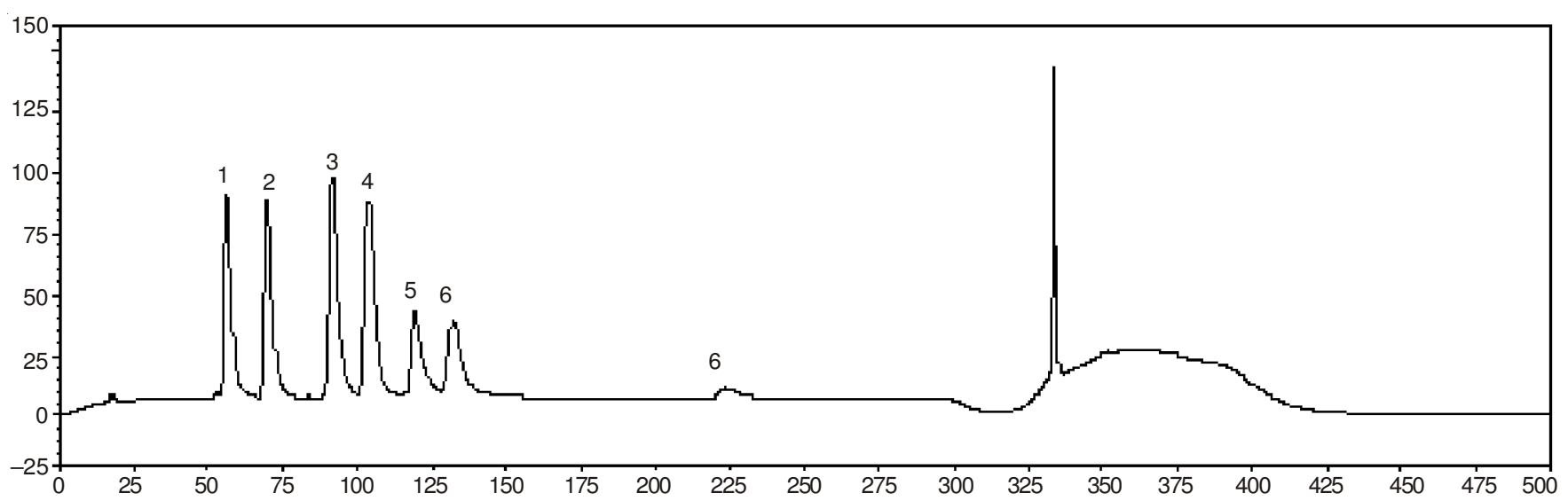

(1) Rhamnose, (2), Arabinose, (3) Glucose, (4) Xylose, (5) Aevulose, (6) Sucrose, (7) Maltose

Fig. 2. Chromatogram of seven standards 


\begin{tabular}{|c|c|c|c|c|c|c|c|c|}
\hline \multicolumn{9}{|c|}{$\begin{array}{c}\text { TABLE-3 } \\
\text { RESULT OF DIFFERENT EXTRACT SOLVENT }\end{array}$} \\
\hline $\begin{array}{l}\text { Extraction } \\
\text { methods }\end{array}$ & Sample & $\begin{array}{l}\text { Rhamnose } \\
(\mathrm{m} \%)\end{array}$ & $\begin{array}{l}\text { Arabinose } \\
(\mathrm{m} \%)\end{array}$ & $\begin{array}{c}\text { Glucose } \\
(\mathrm{m} \%)\end{array}$ & $\begin{array}{l}\text { Xylose } \\
(\mathrm{m} \%)\end{array}$ & $\begin{array}{l}\text { Fructose } \\
(\mathrm{m} \%)\end{array}$ & $\begin{array}{l}\text { Sucrose } \\
(\mathrm{m} \%)\end{array}$ & $\begin{array}{l}\text { Maltose } \\
(\mathrm{m} \%)\end{array}$ \\
\hline $5 \%$ & A & 0.0165 & 0.0628 & 6.9954 & - & 12.1782 & 0.3619 & 0.8307 \\
\hline $\mathrm{CH}_{3} \mathrm{COOH}$ & B & 0.0072 & 0.0709 & 6.6950 & - & 11.5857 & 0.2331 & 1.1159 \\
\hline \multirow{2}{*}{$\mathrm{H}_{2} \mathrm{O}$} & A & 0.0587 & 0.0920 & 7.4677 & - & 13.1186 & 0.3620 & 3.9563 \\
\hline & B & 0.0584 & 0.1127 & 6.9914 & - & 12.1765 & 0.3748 & 3.8204 \\
\hline \multirow{2}{*}{$\begin{array}{l}0.01 \mathrm{mmol} / \mathrm{L} \\
\mathrm{NaOH}\end{array}$} & A & 0.0331 & 0.1025 & 7.2379 & - & 12.6680 & 0.3177 & 3.4303 \\
\hline & $\mathrm{B}$ & 0.0477 & 0.1026 & 6.8305 & - & 11.4806 & 0.2697 & 3.2726 \\
\hline \multicolumn{9}{|c|}{$\begin{array}{l}\text { TABLE-4 } \\
\text { OF SAMPLE VOLUME }\end{array}$} \\
\hline Sample amount & Sample & Rhamnose (\%) & Arabinose $(\%)$ & Glucose (\%) & Xylose (\%) & Fructose $(\%)$ & Sucrose $(\%)$ & Maltose $(\%)$ \\
\hline \multirow{2}{*}{$1 \mathrm{mg} / \mathrm{L}$} & A & 0.0082 & 0.0661 & 8.3593 & - & 14.1673 & 0.3902 & 4.4261 \\
\hline & B & 0.0603 & 0.0764 & 8.2645 & - & 14.2354 & 0.2970 & 4.5012 \\
\hline \multirow{2}{*}{$4 \mathrm{mg} / \mathrm{L}$} & A & 0.0050 & 0.0598 & 7.1639 & - & 11.7388 & 0.2046 & 3.9316 \\
\hline & B & 0.0548 & 0.0561 & 8.0097 & - & 12.2063 & 0.1309 & 3.9528 \\
\hline \multirow{2}{*}{$10 \mathrm{mg} / \mathrm{L}$} & A & 0.0212 & 0.0131 & 0.9638 & - & 1.5491 & 0.0909 & 0.6016 \\
\hline & B & 0.0204 & 0.0129 & 0.9443 & - & 1.5428 & 0.0838 & 0.4686 \\
\hline
\end{tabular}

was added, respectively into six uniform flasks. Aliquots of $50 \mathrm{~mL}$ of ultrapure water were added into these flasks respectively. Secondly, these flasks containing the mixture were under ultrasonic condition for 10, 20, 30, 40, 50 and $60 \mathrm{~min}$, respectively. Finally, after analysis, the results were showed in Table5. It is clear from Table-5 that the amounts of sugar were increased with time within $20 \mathrm{~min}$ and there was no obvious change from 20 to $60 \mathrm{~min}$. Thus, $20 \mathrm{~min}$ was selected as the optimal ultrasonic time.

Selection of ultrasonic extraction power: Accurate weigh $50 \mathrm{mg}$ of the samples with 4 sets. Then $50 \mathrm{~mL}$ ultrapure water was added to each sample and ultrasonic extracted them under the power of 40, 60, 80 and $100 \mathrm{~Hz}$, respectively. Finally carried them on analysis, the result was shown in Table-6. The result shows that the extraction quantity has no significant change when the ultrasonic retraction power is beyond $40 \mathrm{~Hz}$. Therefore $40 \mathrm{~Hz}$ was chosen as the retraction power.

Selection of ultrasonic extraction temperature: Precision weighed $50 \mathrm{mg}$ of the products in 6 sets. Then $50 \mathrm{~mL}$ ultrapure water was added to each sample and ultrasonic extracted them under the temperature of $10,25,40,55,70$ and $80^{\circ} \mathrm{C}$, respectively. Finally carried them on analysis, the result is shown in Table-7. We can see from the result that the extraction yield of sugar increases with the rising of temperature when below $25^{\circ} \mathrm{C}$. There is a little effects on the extraction yield of sugars when the temperature is between 25 and $55^{\circ} \mathrm{C}$. Fructose and

TABLE-5

RESULTS OF SAMPLE EXTRACTION TIME

\begin{tabular}{cccccccc}
\hline Ultrasonic time (min) & Rhamnose (\%) & Arabinose (\%) & Glucose (\%) & Xylose (\%) & Fructose (\%) & Sucrose (\%) & Maltose (\%) \\
\hline 10 & 0.0234 & 0.0664 & 4.4219 & - & 9.0005 & 0.3444 & 1.6180 \\
20 & 0.0287 & 0.0864 & 4.5488 & - & 9.6511 & 0.5915 & 1.9385 \\
30 & 0.0289 & 0.0863 & 4.5801 & - & 9.6919 & 0.5933 & 1.9072 \\
40 & 0.0288 & 0.0864 & 4.5662 & - & 9.7393 & 0.5705 & 1.9442 \\
50 & 0.0289 & 0.0855 & 4.5082 & - & 9.7916 & 0.5521 & 1.9695 \\
60 & 0.0290 & 0.0875 & 4.5677 & - & 9.7966 & 0.5964 & 1.9728 \\
\hline
\end{tabular}

TABLE-6

RESULTS OF ULTRASONIC POWER

\begin{tabular}{cccccccc}
\hline Ultrasonic power $(\mathrm{Hz})$ & Rhamnose $(\%)$ & Arabinose $(\%)$ & Glucose $(\%)$ & Xylose $(\%)$ & Fructose $(\%)$ & Sucrose $(\%)$ & Maltose $(\%)$ \\
\hline 40 & 0.0226 & 0.0884 & 5.1398 & - & 10.9181 & 0.7976 & 2.6669 \\
60 & 0.0221 & 0.0887 & 5.1793 & - & 10.9227 & 0.8001 & 2.6671 \\
80 & 0.0231 & 0.0888 & 5.1982 & - & 11.0104 & 0.8078 & 2.7014 \\
100 & 0.0232 & 0.0890 & 5.1655 & - & 11.0422 & 0.8118 & 2.7169 \\
\hline
\end{tabular}

TABLE-7

RESULTS OF EXTRACTION TEMPERATURE

\begin{tabular}{|c|c|c|c|c|c|c|c|}
\hline Ultrasonic temperature $\left({ }^{\circ} \mathrm{C}\right)$ & Rhamnose (\%) & Arabinose $(\%)$ & Glucose $(\%)$ & Xylose $(\%)$ & Fructose $(\%)$ & Sucrose $(\%)$ & Maltose $(\%)$ \\
\hline 10 & 0.0100 & 0.0578 & 5.2506 & - & 9.9244 & 0.5126 & 2.7484 \\
\hline 25 & 0.0105 & 0.0652 & 5.4088 & - & 10.0891 & 0.5526 & 2.8207 \\
\hline 40 & 0.0106 & 0.0668 & 5.4858 & - & 10.0990 & 0.5420 & 2.8183 \\
\hline 55 & 0.0107 & 0.0669 & 5.4646 & - & 10.1471 & 0.5366 & 2.7070 \\
\hline 70 & 0.0109 & 0.0681 & 5.5581 & - & 10.1946 & 0.4855 & 2.5107 \\
\hline 80 & 0.0110 & 0.0697 & 5.6153 & - & 10.2535 & 0.4453 & 2.4916 \\
\hline
\end{tabular}


maltose have been breakdown when the temperature beyond $55^{\circ} \mathrm{C}$. Hence, $55^{\circ} \mathrm{C}$ was selected as the ultrasonic extraction temperature.

Selection of pre-treatment method for sample column: It turned out that the obtained solution became darker after the pre-treatment of the sample, which can be demonstrated that the sample solution contains a lot of pigments and macromolecules extracted from tobacco. In order to prevent these substances interfere with the determination of sugars and cause damage to the chromatography column to shorten its lifetime, the recommended method of Dionex OnGuard II RP was produced to filter the samples by the pretreatment cartridge. Therefore, the samples before and after filtered were analyzed. It turned out that the pigments and other macromolecules in the samples have little effects on measurement. Furthermore, the column performance changed little compared with the previous testing samples without using of the OnGuard II RP sample preparation cartridge. Therefore samples are only filtered by $0.45 \mu \mathrm{m}$ nylon membrane before injection.

Determination methods: The optimum experimental conditions are selected finally through a large number of optimization experiments which carried out under conditions of method validation, including the linear relationship, the detection limit, precision, stability, repeatability and the determination of the recovery.

Determination of the linear relationship with the detection limit: Preparation of the standard curve: The precise amount of $0.01,0.05,0.10,0.25,0.50,0.75$ and $1 \mathrm{~mL}$ mixed standard solution $(2 \mathrm{mg} / \mathrm{mL})$ are measured in seven $10 \mathrm{~mL}$ volumetric flask, adding $5 \mathrm{mmol} / \mathrm{L}$ sodium hydroxide to volume, and the mixed solution was obtained under the conditions shown in the Table-2. On analysis of the linear regression, the concentration of each sugar solution is used as abscissa, the peak area of each sugar is used as ordinate. Linear regression equation, linear range and detection limit of various sugars are obtained (Table-8).

\begin{tabular}{|c|c|c|c|}
\hline \multicolumn{4}{|c|}{$\begin{array}{c}\text { TABLE-8 } \\
\text { REGRESSION EQUATIONS, CORRELATION } \\
\text { COEFFICIENTS AND LIMIT OF DETECTION }\end{array}$} \\
\hline Sample & $\begin{array}{l}\text { Equation of linear regression } \\
\qquad \mathrm{R}^{2}(\%)\end{array}$ & $\begin{array}{l}\text { Linear } \\
\text { range } \\
(\mu \mathrm{g} / \mathrm{mL})\end{array}$ & $\begin{array}{l}\text { LODs } \\
(\mu \mathrm{g} / \mathrm{mL})\end{array}$ \\
\hline Rhamnose & $\begin{array}{c}Y=1.9610 X-0.0961 \\
R^{2}=99.9356\end{array}$ & $2-200$ & 0.0002 \\
\hline Arabinose & $\begin{array}{c}Y=1.9585 X+1.1958 \\
R^{2}=99.9492\end{array}$ & $2-200$ & 0.0002 \\
\hline Glucose & $\begin{array}{c}\mathrm{Y}=3.7484 \mathrm{X}+0.6803 \\
\mathrm{R}^{2}=99.9461\end{array}$ & $2-200$ & 0.0002 \\
\hline Xylose & $\begin{array}{c}\mathrm{Y}=2.2274 \mathrm{X}-1.6835 \\
\mathrm{R}^{2}=99.9764\end{array}$ & $2-200$ & 0.0002 \\
\hline Fructose & $\begin{array}{c}Y=1.3559 X-0.0798 \\
R^{2}=99.9978\end{array}$ & $2-200$ & 0.0004 \\
\hline Sucrose & $\begin{array}{c}Y=1.5408 X-1.4422 \\
R^{2}=99.9662\end{array}$ & $2-200$ & 0.0003 \\
\hline Maltose & $\begin{array}{c}Y=0.2477 X-0.3038 \\
R^{2}=99.9561\end{array}$ & $2-200$ & 0.0005 \\
\hline
\end{tabular}

\section{Precision, repeatability and stability testing}

Determination of precision: The same standard sample solution (concentration) were repeated 10 times. The peak area relative standard deviation (RSD) of rhamnose, arabinose, glucose, xylose, fructose, sucrose and maltose were $2.7405,2.5440$, 5.8697, 3.2873, 5.6146, 3.2831 and $2.2803 \%$, respectively.

Repeatability determination: Samples were pre-treated by the optimized pretreatment and analyzed by the optimized analytical conditions (sample parallel determinations for seven times). The peak area relative standard deviation (RSD) of rhamnose, arabinose, glucose, xylose, fructose, sucrose, maltose peak area relative standard deviation (RSD) were 4.1405, 5.4616, 0.5222, 1.5275, 2.4502, 3.3375 and $5.0116 \%$.

Determination of stability: Taken a standard sample (concentration) to save in a refrigerator at $4{ }^{\circ} \mathrm{C}$ for $0,2,6,12$ and $24 \mathrm{~h}$ and then tested. The peak area relative standard deviation (RSD) rhamnose, arabinose, glucose, xylose, fructose, sucrose, maltose were 3.7169, 4.5011, 1.7826, 0.6350, 2.7195, $4.1735,2.0437 \%$, The data shows that the tested substance were stable within $24 \mathrm{~h}$.

Recovery determination: The recovery is determined by standard addition method in this study. The three same samples were weighed to test the content of sugar of the samples containing added sugar and the sample without containing added sugar by adding a certain amount of standard samples of sugar. The results are shown in Table-9. The recoveries are between 95 and $105 \%$.

\begin{tabular}{|c|c|c|c|c|}
\hline \multicolumn{5}{|c|}{$\begin{array}{c}\text { TABLE-9 } \\
\text { RESULTS OF THE RECOVERY EXPERIMENTS }\end{array}$} \\
\hline Samples & $\begin{array}{l}\text { Sample amount } \\
(\mu \mathrm{g} / \mathrm{mg})\end{array}$ & $\begin{array}{c}\text { Addition amount } \\
(\mu \mathrm{g} / \mathrm{mg})\end{array}$ & $\begin{array}{c}\text { Measured } \\
\text { amount }(\mu \mathrm{g} / \mathrm{mg})\end{array}$ & $\begin{array}{c}\text { Recovery } \\
(\%)\end{array}$ \\
\hline \multirow{3}{*}{ Rhamnose } & \multirow{3}{*}{0.0000} & 0.3000 & 0.2981 & 99.3500 \\
\hline & & 0.6000 & 0.5816 & 96.9250 \\
\hline & & 1.2000 & 1.2420 & 103.4958 \\
\hline \multirow{3}{*}{ Arabinose } & \multirow{3}{*}{0.4226} & 0.3000 & 0.7148 & 97.4000 \\
\hline & & 0.6000 & 1.0415 & 103.1500 \\
\hline & & 1.2000 & 1.6003 & 98.1417 \\
\hline \multirow{3}{*}{ Glucose } & \multirow{3}{*}{58.7549} & 32.5000 & 91.5966 & 101.0515 \\
\hline & & 65.0000 & 123.0270 & 98.8802 \\
\hline & & 130.0000 & 187.4532 & 98.9987 \\
\hline \multirow{3}{*}{ Xylose } & \multirow{3}{*}{0.0000} & 0.3000 & 0.3107 & 103.5667 \\
\hline & & 0.6000 & 0.5785 & 96.4167 \\
\hline & & 1.2000 & 1.2552 & 104.6000 \\
\hline \multirow{3}{*}{ Fructose } & \multirow{3}{*}{110.7904} & 57.5000 & 168.0473 & 99.5771 \\
\hline & & 115.0000 & 225.4038 & 99.6638 \\
\hline & & 230.0000 & 343.9179 & 101.3598 \\
\hline \multirow{3}{*}{ Fructose } & \multirow{3}{*}{18.2647} & 2.5000 & 20.8767 & 104.4800 \\
\hline & & 5.0000 & 23.4186 & 103.0780 \\
\hline & & 10.0000 & 28.4818 & 102.1710 \\
\hline \multirow{3}{*}{ Maltose } & \multirow{3}{*}{8.9917} & 8.0000 & 16.9209 & 99.1150 \\
\hline & & 16.0000 & 24.8369 & 99.0325 \\
\hline & & 32.0000 & 41.5480 & 101.7383 \\
\hline
\end{tabular}

Sample analysis: The samples were measured via the experimental methods and conditions described above (sample obtained from Hunan tobacco industry limited liability company) to obtain a sample chromatogram shown in Fig. 3. The spectrum (Table-10) shows that there is not interference between each peak, which indicates that the method is specific.

Measurements were performed on dozens of cigarette samples and the results were showed in Table-10. Compared with HPLC-RI method and mass spectrometry which are commonly used in sugar measuring, we found that the three results were basically consistent. Furthermore, the most 
CONTENTS OF SEVEN KINDS OF CARBOHYDRATES IN TOBACCOS

\begin{tabular}{|c|c|c|c|c|c|c|c|c|c|}
\hline S. N. & Sample & Rhamnose (\%) & Arabinose (\%) & Glucose (\%) & Xylose (\%) & Fructose $(\%)$ & Sucrose $(\%)$ & Maltose (\%) & Total content (\%) \\
\hline 1 & LinhaiLingzhi & - & 0.0010 & 2.2484 & - & 4.2866 & 1.0503 & 0.1404 & 7.7266 \\
\hline 2 & Jinqiao A & 0.0003 & 0.0019 & 2.7183 & - & 6.0303 & 0.8956 & 0.1169 & 9.7631 \\
\hline 3 & Baisha B & 0.0047 & 0.0229 & 3.6066 & - & 6.9052 & 0.8078 & 0.2171 & 11.5643 \\
\hline 5 & Zhongnanhai & 0.0019 & 0.0193 & 3.0924 & - & 7.0707 & 0.9180 & 0.1141 & 11.2164 \\
\hline 6 & Jinqiao B & - & 0.0121 & 3.1586 & - & 8.3407 & 0.2560 & 0.0000 & 11.7674 \\
\hline 7 & Baisha C & - & 0.0042 & 6.4021 & - & 11.4921 & 0.5200 & 0.3169 & 18.7353 \\
\hline 8 & Baisha D & - & 0.0097 & 5.8088 & - & 9.9851 & 1.4485 & 0.4328 & 17.6849 \\
\hline 9 & Baisha E & - & 0.0081 & 6.0234 & - & 10.0228 & 1.2864 & 0.2699 & 17.6106 \\
\hline 10 & Baisha F & - & 0.0066 & 6.6967 & - & 10.1117 & 2.4009 & 0.4110 & 19.6269 \\
\hline 11 & Baisha G & 0.0015 & 0.0048 & 6.9572 & - & 10.6422 & 2.2153 & 0.3291 & 20.1501 \\
\hline 12 & Baisha H & 0.0012 & 0.0056 & 7.1501 & - & 11.8259 & 1.6993 & 0.3091 & 20.9912 \\
\hline 13 & Baisha I & 0.0012 & 0.0021 & 5.1655 & - & 10.3374 & 0.6768 & 0.0985 & 16.2815 \\
\hline 14 & Baisha J & - & 0.0094 & 5.2012 & - & 10.0236 & 0.9227 & 0.2751 & 16.4320 \\
\hline 15 & Wuhuashen & 0.0016 & 0.0010 & 7.3045 & - & 10.3976 & 0.4843 & 0.1683 & 18.3573 \\
\hline 16 & Shuangxi & 0.0004 & 0.0015 & 4.8770 & - & 8.1609 & 0.6786 & 0.0851 & 13.8035 \\
\hline 18 & Hongqi Canal & 0.0001 & 0.0019 & 6.3745 & - & 9.4758 & 0.6859 & 0.1326 & 16.6708 \\
\hline 19 & Dihao & 0.0001 & 0.0036 & 4.8298 & - & 8.4798 & 0.6948 & 0.0933 & 14.1013 \\
\hline 20 & Jinsheng A & 0.0004 & - & 6.2358 & - & 10.1269 & 0.7900 & 0.1211 & 17.2741 \\
\hline 21 & Jinsheng B & - & 0.0021 & 8.6169 & - & 12.2876 & 1.2205 & 0.1331 & 22.2603 \\
\hline 22 & Yunyan & 0.0001 & 0.0088 & 11.2988 & 0.0029 & 13.9158 & 2.3171 & 0.2108 & 27.7541 \\
\hline 23 & Lanzhou & 0.0008 & - & 12.8850 & - & 15.1875 & 3.7299 & 0.3299 & 32.1330 \\
\hline 24 & Changbaishan A & - & - & 11.5276 & 0.0010 & 21.7903 & 1.3046 & 0.1919 & 34.8154 \\
\hline 25 & Changbaishan B & 0.0010 & - & 7.8750 & 0.0015 & 15.4464 & 1.6950 & 0.0751 & 25.0940 \\
\hline 26 & Zhonghua A & - & 0.0033 & 10.7700 & 0.0264 & 13.6669 & 1.9290 & 0.6818 & 27.0773 \\
\hline 27 & Zhonghua B & 0.0015 & 0.0031 & 9.1881 & - & 14.2071 & 1.0054 & 0.2413 & 24.6465 \\
\hline 28 & Hongshuangxi & 0.0013 & 0.0049 & 6.8666 & - & 17.1376 & 0.6230 & 0.0426 & 24.6760 \\
\hline 29 & Furongwang A & - & - & 7.6633 & - & 9.8855 & 1.4654 & 0.2681 & 19.2823 \\
\hline 30 & Furongwang B & 0.0028 & 0.0029 & 8.6966 & - & 12.4913 & 3.1176 & 0.6324 & 24.9435 \\
\hline 31 & Baisha K & 0.0013 & 0.0015 & 4.6681 & - & 7.3629 & 0.8446 & 0.2280 & 13.1064 \\
\hline 32 & Baisha L & 0.0011 & - & 5.0584 & - & 7.9193 & 0.7754 & 0.1393 & 13.8934 \\
\hline 33 & Guiyan & 0.0011 & 0.0011 & 5.7821 & - & 8.3783 & 0.7383 & 0.1836 & 15.0845 \\
\hline 36 & Yunyan B & 0.0006 & - & 6.8241 & - & 8.7968 & 1.6464 & 0.1275 & 17.3954 \\
\hline 37 & Honghe & 0.0006 & - & 5.2966 & - & 8.5393 & 0.9120 & 0.1593 & 14.9078 \\
\hline 38 & Huanghelou A & 0.0003 & 0.0019 & 5.8888 & - & 8.5441 & 0.8640 & 0.1163 & 15.4153 \\
\hline 39 & Huanghelou B & - & 0.0019 & 6.6444 & - & 9.3543 & 0.6176 & 0.1641 & 16.7823 \\
\hline 40 & GoldenDragon & - & - & 5.9124 & - & 8.6186 & 0.5889 & 0.1705 & 15.2904 \\
\hline 41 & Diamond A & 0.0006 & 0.0034 & 5.7313 & - & 9.4365 & 0.8855 & 0.0931 & 16.1504 \\
\hline 42 & Diamond B & 0.0011 & 0.0014 & 5.7845 & - & 9.1988 & 0.5758 & 0.1438 & 15.7053 \\
\hline 43 & Yuxi & - & - & 6.1594 & - & 9.8891 & 2.2179 & 0.2276 & 18.4940 \\
\hline 44 & Hongtashan & 0.0003 & - & 6.1835 & - & 8.6791 & 1.6526 & 0.1799 & 16.6954 \\
\hline 45 & Huangshan A & - & 0.0031 & 5.3488 & - & 8.3956 & 0.4044 & 0.1749 & 14.3268 \\
\hline 46 & Huangshan B & 0.0044 & - & 4.4090 & - & 16.8521 & 0.8721 & 0.1774 & 22.3150 \\
\hline 47 & Liqun A & 0.0019 & 0.0029 & 6.4408 & - & 9.4736 & 0.9264 & 0.1593 & 17.0048 \\
\hline 48 & Liqun B & 0.0018 & 0.0026 & 6.1958 & - & 9.0505 & 0.7470 & 0.1755 & 16.1731 \\
\hline 49 & Dahongying & 0.0013 & 0.0040 & 5.2355 & - & 8.0613 & 0.9643 & 0.1333 & 14.3995 \\
\hline 50 & General & - & 0.0015 & 4.5948 & - & 7.3829 & 1.2758 & 0.0881 & 13.3430 \\
\hline 51 & Taishan & 0.0003 & 0.0013 & 7.6416 & - & 9.6944 & 1.6700 & 0.1841 & 19.1916 \\
\hline 52 & Zhenlong & 0.0006 & 0.0013 & 6.8996 & - & 9.5141 & 0.9134 & 0.1885 & 17.5175 \\
\hline 53 & Suyan & 0.0005 & 0.0011 & 6.8876 & - & 9.4370 & 0.8075 & 0.1465 & 17.2803 \\
\hline 54 & Nanjing & 0.0009 & 0.0016 & 5.5020 & - & 8.0401 & 0.8429 & 0.1574 & 14.5449 \\
\hline 55 & Yipinmei & - & 0.0009 & 5.2393 & - & 8.0923 & 1.2786 & 0.1586 & 14.7696 \\
\hline 56 & Pride A & 0.0009 & 0.0023 & 5.6404 & - & 8.8501 & 0.7835 & 0.1284 & 15.4055 \\
\hline 57 & Pride B & 0.0035 & - & 5.8843 & - & 15.0018 & - & - & 20.8895 \\
\hline 58 & Longfeng & 0.0006 & - & 5.4824 & - & 9.3351 & 0.4710 & 0.1930 & 15.4821 \\
\hline 59 & Haomao & 0.0006 & 0.0010 & 7.0515 & - & 11.1028 & 0.9795 & 0.1924 & 19.3278 \\
\hline 60 & Houwang & - & 0.0018 & 4.6733 & - & 8.6399 & 1.3200 & 0.1110 & 14.7459 \\
\hline 61 & 09X2F Huaihua & 0.0005 & - & 7.2630 & - & 10.1111 & 1.5574 & 0.1415 & 19.0735 \\
\hline 62 & 09X2F Sangzhi & - & 0.0074 & 5.7954 & - & 9.7071 & 3.7714 & 0.0443 & 19.3255 \\
\hline 63 & B3F Longshan & - & 0.0019 & 4.5311 & - & 7.3505 & 1.7159 & 0.0746 & 13.6740 \\
\hline 64 & 08C 2 F Chuxiong & 0.0009 & 0.0066 & 9.8568 & - & 11.0859 & 1.6233 & 0.2544 & 22.8278 \\
\hline 65 & C2F Yuanjiang & 0.0004 & - & 5.4015 & - & 8.3333 & 0.5761 & 0.1410 & 14.4523 \\
\hline 66 & Zimbabwe & 0.0014 & 0.0135 & 4.8476 & - & 9.2219 & 0.5039 & 0.2388 & 14.8270 \\
\hline 67 & Top cigarette & - & 0.0063 & 6.1375 & - & 11.1278 & 2.4940 & 0.3494 & 20.1150 \\
\hline
\end{tabular}


sensitive method is ion chromatography, whose measurement results were slightly higher than refractive index and similar with mass spectrometry. Ion chromatography can detect the presence of small amounts of rhamnose, arabinose and xylose while refractive index method cannot detect. The results of ion chromatography are more accurate and reliable. Ion chromatography operation less depend on the environment, which is easy to operate, friendly to environmental and more convenient than the refractive index and mass spectrometry method.
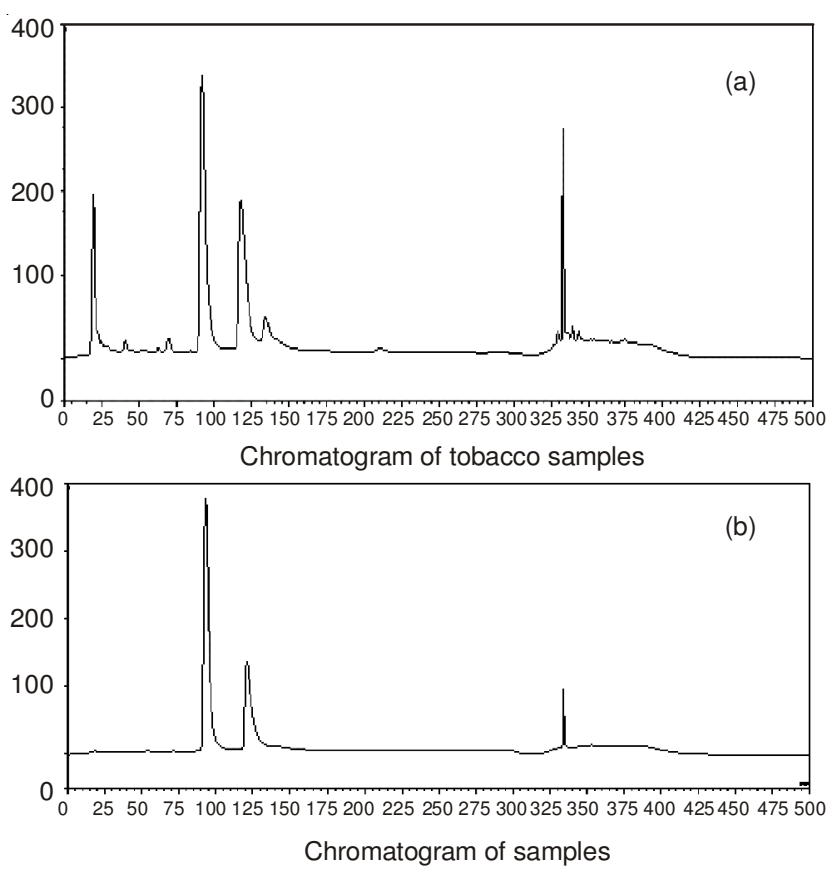

Fig. 3. Chromatogram of samples

As shown in Fig. 4, the total contents of seven kinds of sugars of different kinds tobacco samples are distributed in 5 to $35 \%$, mainly concentrated in 15 to $25 \%$, which are accorded with the carbohydrate content of cigarettes. Furthermore, the amount of cigarettes whose total contents of seven kinds of sugars are below the total range of 15 to $25 \%$ is relatively small, including Baisha, Marlboro, Zhongnanhai, Jinqiao and woodlands fungus. It can be found that these samples are mixed in cigarette smoke. Hybrid cigarette use various tobacco beside tobacco, there are Maryland tobacco smoke, white smoke aid, spices and smoke, in which a variety of other sugar were lower than the smoke of tobacco, thus come to the result that the mixed tobacco has lower sugar content. And the concentrated at 15 to $25 \%$ of the finished product are tobacco smoke, the smoke Wherein there are several high sugar content, which is in relation to the origin of the additives in tobacco. The upper,

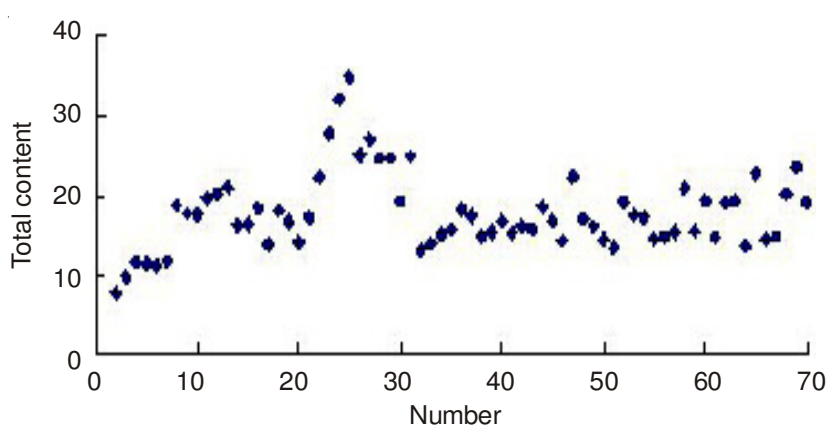

Fig. 4. Content of total sugars in the samples

middle and lower part of sugar in smoke are in a certain regularity situation in which the central smoke is highest, lower smoke is the minimum. Taking the tobacco of Huaihua, Sangzhi, Yongsan, Chuxiong, Yuanjiang and other parts into comparison, we can see the total sugar content of Longshan is low, which is related to the environment of tobacco growing regions.

\section{Conclusion}

A simple and reproducible method was established for the detection the carbohydrates of cigarettes. Following a simple water extraction from tobacco, the carbohydrates are separated using high-performance anion exchange chromatography and directly detected using pulsed amperometric detection. Furthermore, it demonstrated that HPAEC-PAD is high sensitive and selective, convenient, low-cost and applicative tool for analyses of carbohydrates in tobacco products. Application of described method allows the determination of carbohydrates whose evaluation could be a useful process to control and process.

\section{REFERENCES}

1. J.R. Shifflett, L.A. Jones, E.R. Limowski and D.Z. Bezabeh, J. Agric. Food Chem., 60, 11714 (2012).

2. S.W. Purkis, C. Mueller and M. Intorp, Food Chem. Toxicol., 49, 3238 (2011).

3. R. Talhout, A. Opperhuizen and J.G.C. van Amsterdam, Food Chem. Toxicol., 44, 1789 (2006).

4. R.K. Sharma, J.B. Wooten, V.L. Baliga, P.A. Martoglio-Smith and M.R. Hajaligol, J. Agric. Food Chem., 50, 771 (2002).

5. F.N. Lamari, R. Kuhn and N.K. Karamanos, J. Chromatogr. B, 793, 15 (2003).

6. C.M. Zook, P.M. Patel, W.R. LaCourse and S. Ralapati, J. Agric. Food Chem., 44, 1773 (1996).

7. M. Raessler, TrAC Trends Anal. Chem., 30, 1833 (2011).

8. K. Kaiser and R. Benner, Anal. Chem., 72, 2566 (2000).

9. G. Arfelli and E. Sartini, Food Chem., 142, 152 (2014).

10. S.C. Hsu, R.L. Pollack, A.F. Hsu and R.E. Going, J. Am. Dent. Assoc., 101, 915 (1980). 